\title{
Continuous interval exchange actions
}

\author{
CHRISTOPHER F NOVAK
}

Let $\mathcal{E}$ denote the group of all interval exchange transformations on $[0,1)$. Given a suitable topological group structure on $\mathcal{E}$, it is possible to classify all one-parameter interval exchange actions (continuous homomorphisms $\mathbb{R} \rightarrow \mathcal{E}$ ). In particular, up to conjugacy in $\mathcal{E}$, any one-parameter interval exchange action factors through a rotational torus action.

37E05, 54H15; 57S05, 37A10, 57M60

\section{Introduction}

An interval exchange transformation is a map $[0,1) \rightarrow[0,1)$ defined by a finite partition of the unit interval into half-open intervals and a rearrangement of these intervals by translation. See Figure 1 for a graphical example.

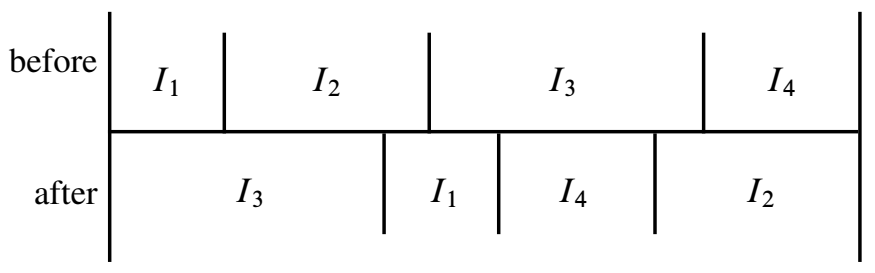

Figure 1: An interval exchange with $\pi=\left(\begin{array}{llll}1 & 2 & 4 & 3\end{array}\right)$

The dynamics of interval exchanges were first studied in the late seventies by Keane [9; 10], Katok [8], Rauzy [16], Veech [17] and others. This initial stage of research culminated in the independent proofs by Masur [12] and Veech [18] that almost every interval exchange is uniquely ergodic. See the recent survey of Viana [19] for a unified presentation of these results.

There is currently much interest and activity in the dynamics of interval exchanges. This is due in part to the recent resolution of certain long-standing problems in this area; one important example is the work of Avila and Forni [1] in which they prove that almost every interval exchange is weakly mixing. Much of the study of interval 
exchanges is closely related to dynamics on the moduli space of translation surfaces; an introduction to this topic and its connection to interval exchanges is found in a survey of Zorich [20].

An extension of the study of single interval exchanges is to consider their dynamics in terms of group actions. The set $\mathcal{E}$ of all interval exchange transformations forms a group under composition, and an interval exchange action of a group $G$ is a homomorphism $G \rightarrow \mathcal{E}$. A general and fundamental question is to determine if a given group $G$ has faithful interval exchange actions. More broadly, it can be asked if there are general algebraic obstructions to the existence of such actions. On the other hand, if such actions do exist for a given group, it is desirable to attempt to classify them in some way. The goal of this paper is to classify continuous interval exchange actions of the group $\mathbb{R}$.

The study of interval exchange actions is motivated by the study of other transformation groups, particularly groups of homeomorphisms and diffeomorphisms of onedimensional manifolds. However, what is known about the structure of $\mathcal{E}$ suggests that there may be substantial differences between $\mathcal{E}$ and these groups. For instance, the author showed in [15] that no subgroup of $\mathcal{E}$ has distortion elements. In contrast, the groups $\operatorname{Diff}^{\omega}(\mathbb{R})$ and Diff ${ }^{\omega}\left(S^{1}\right)$ of real-analytic diffeomorphisms on the line and circle both contain such elements. See Franks [5] for definitions, examples and results involving actions of groups having distortion elements.

In addition, many basic questions that are well understood for diffeomorphisms of 1 -manifolds are currently open for the group $\mathcal{E}$. For instance:

(1) Does $\mathcal{E}$ contain a free subgroup on two generators? (Katok)

(2) Does $\mathcal{E}$ contain groups of intermediate growth? (Grigorchuk)

(3) Is every solvable subgroup of $\mathcal{E}$ virtually abelian? (Navas)

For question (1), it is easy to construct examples of nonabelian free groups in $\operatorname{Diff}\left(S^{1}\right)$ or $\operatorname{Diff}(\mathbb{R})$ by means of the ping-pong construction. More detailed results, analogous to the Tits' alternative, are also known for $\operatorname{Homeo}_{+}\left(S^{1}\right)$ and $\operatorname{Diff}^{\omega}\left(S^{1}\right)$; see Margulis [11] and Farb and Shalen [4], respectively. Question (2) is answered in the affirmative for the group $\operatorname{Diff}_{+}^{1}([0,1])$ by Navas [14]. This work also shows that for any $\alpha>0$, any subgroup of $\operatorname{Diff}_{+}^{1+\alpha}([0,1])$ with subexponential growth must be virtually nilpotent. This gives a negative answer to question (2) in this case, due to the fundamental result of Gromov [6] that the finitely generated virtually nilpotent groups are exactly those having polynomial growth. Question (3) is also well understood for various transformation groups on 1-manifolds; for instance, see Bleak [2], Burslem and Wilkinson [3] and Navas [13]. 
To introduce the results of the current work, consider the following precise definition and notation for an interval exchange. Let $\pi \in \Sigma_{n}$ be a permutation of $\{1,2, \ldots, n\}$, and let $\lambda$ be a vector in the simplex

$$
\Lambda_{n}=\left\{\lambda=\left(\lambda_{1}, \ldots, \lambda_{n}\right): \lambda_{i}>0, \sum \lambda_{i}=1\right\} \subseteq \mathbb{R}^{n}
$$

The vector $\lambda$ induces a partition of $[0,1)$ into intervals

$$
I_{j}=\left[\beta_{j-1}:=\sum_{i=1}^{i=j-1} \lambda_{i}, \quad \beta_{j}:=\sum_{i=1}^{i=j} \lambda_{i}\right), \quad 1 \leq j \leq n .
$$

Let $f_{(\pi, \lambda)}$ be the interval exchange that translates each $I_{j}$ such that the ordering of these intervals within $[0,1)$ is permuted according to $\pi$. More precisely,

$$
f_{(\pi, \lambda)}(x)=x+\omega_{j} \quad \text { if } x \in I_{j},
$$

where

$$
\omega_{j}=\Omega_{\pi}(\lambda)_{j}=\sum_{i: \pi(i)<\pi(j)} \lambda_{i}-\sum_{i: i<j} \lambda_{i}
$$

Note that $\Omega_{\pi}: \Lambda_{n} \rightarrow \mathbb{R}^{n}$ is a linear map depending only on $\pi$.

An initial example of an interval exchange $\mathbb{R}$-action is defined by

$$
t \mapsto r_{t},
$$

where $r_{t} \in \mathcal{E}$ is the rotation $r_{t}(x)=x+t(\bmod 1)$, as depicted in Figure 2. This action is not faithful, but its kernel is a discrete subgroup of $\mathbb{R}$.

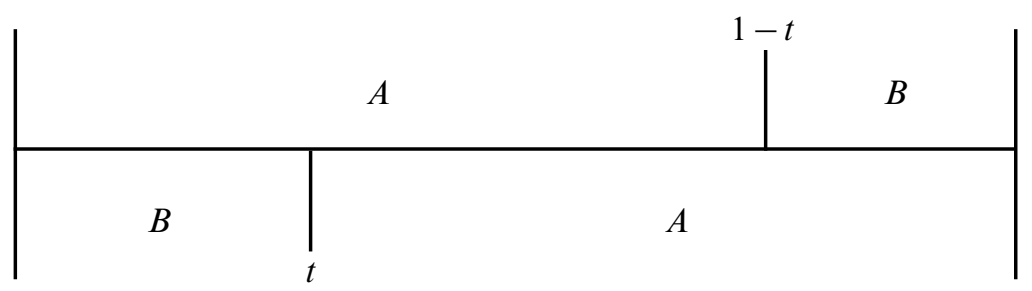

Figure 2: The rotation $r_{t}$ with $\pi=(21)$ and $\lambda=(1-t, t)$

To construct a faithful interval exchange action of $\mathbb{R}$, choose numbers $\alpha_{1}$ and $\alpha_{2}$ in $(0,1)$ such that $\alpha_{1} / \alpha_{2}$ is irrational. For a real number $t$, define $f_{t}$ as the map that 
rotates by $t \alpha_{1}(\bmod 1 / 2)$ on the interval $[0,1 / 2)$ and rotates by $t \alpha_{2}(\bmod 1 / 2)$ on the interval $[1 / 2,1)$. That is, $f_{t}=f_{(\pi, \lambda(t))}$ for the data

$$
\pi=\left(\begin{array}{ll}
1 & 2
\end{array}\right)(34), \quad \lambda(t)=\left(\frac{1-\left\{t \alpha_{1}\right\}}{2}, \frac{\left\{t \alpha_{1}\right\}}{2}, \frac{1-\left\{t \alpha_{2}\right\}}{2}, \frac{\left\{t \alpha_{2}\right\}}{2}\right),
$$

where $\{\cdot\}$ denotes the fractional part of a real number. It is easy to check that $t \mapsto f_{t}$ is a group homomorphism $\mathbb{R} \rightarrow \mathcal{E}$, and the action is faithful due to the assumption that $\alpha_{1} / \alpha_{2}$ is irrational.

This faithful $\mathbb{R}$-action can be viewed as the restriction of an action of the torus $\mathbb{T}^{2}=$ $\mathbb{R}^{2} / \mathbb{Z}^{2}$ (identified with $\left.[0,1) \times[0,1)\right)$ defined by the map $\left(\alpha_{1}, \alpha_{2}\right) \mapsto f_{\left(\pi, \lambda\left(\alpha_{1}, \alpha_{2}\right)\right)}$, where

$$
\pi=(12)(34), \quad \lambda\left(\alpha_{1}, \alpha_{2}\right)=\left(\frac{1-\alpha_{1}}{2}, \frac{\alpha_{1}}{2}, \frac{1-\alpha_{2}}{2}, \frac{\alpha_{2}}{2}\right) .
$$

See Figure 3 for an illustration of the map $f_{\left(\pi, \lambda\left(\alpha_{1}, \alpha_{2}\right)\right)}$.

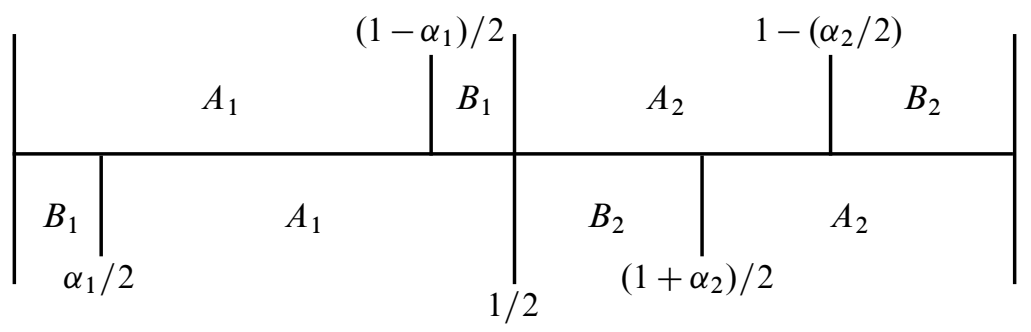

Figure 3: The action $\left(\alpha_{1}, \alpha_{2}\right) \mapsto f_{\left(\pi, \lambda\left(\alpha_{1}, \alpha_{2}\right)\right)}$

A general class of torus actions can be defined by a similar construction. For any partition vector $\lambda \in \Lambda_{n}$ and for any $\alpha=\left(\alpha_{1}, \alpha_{2}, \ldots, \alpha_{n}\right) \in \mathbb{T}^{n}$, define the interval exchange $f_{(\alpha, \lambda)}$ by

$$
f_{(\alpha, \lambda)}: x \mapsto \begin{cases}x+\lambda_{j} \alpha_{j}, & x \in\left[\beta_{j-1}, \beta_{j}-\lambda_{j} \alpha_{j}\right), \\ x+\lambda_{j} \alpha_{j}-\lambda_{j}, & x \in\left[\beta_{j}-\lambda_{j} \alpha_{j}, \beta_{j}\right),\end{cases}
$$

where the points $\beta_{j}$ are the boundary points of the partition intervals defined by $\lambda$. The map $f_{(\alpha, \lambda)}$ is also defined by the permutation $\pi=(12)(34) \cdots((2 n-1) 2 n)$ and the partition vector

$$
\left(\lambda_{1}\left(1-\alpha_{1}\right), \alpha_{1} \lambda_{1}, \lambda_{2}\left(1-\alpha_{2}\right), \alpha_{2} \lambda_{2}, \ldots, \lambda_{n}\left(1-\alpha_{n}\right), \alpha_{n} \lambda_{n}\right) .
$$

The action $\alpha \in \mathbb{T}^{n} \mapsto f_{(\alpha, \lambda)}$ is called the standard torus action associated to $\lambda$. Restricting a standard torus action to a one-parameter subgroup gives an action of $\mathbb{R}$. A (one-parameter) rotation action is defined as any action $\mathbb{R} \rightarrow \mathcal{E}$ that is conjugate 
in $\mathcal{E}$ to a one-parameter subgroup of a standard torus action. The image in $\mathcal{E}$ of a rotation action will be referred to as a rotation subgroup.

The main result of this paper is that under some natural and unrestrictive topological assumptions, the rotation actions classify all continuous interval exchange actions of $\mathbb{R}$. To specify these conditions, define a permutation $\pi \in \Sigma_{n}$ to be unpartitioned if $\pi(j+1) \neq \pi(j)+1$, for all $j$ such that $1 \leq j \leq n-1$. It is shown in Proposition 2.2 that an interval exchange is defined by a unique pair $(\pi, \lambda)$ if one restricts to unpartitioned permutations. For each unpartitioned $\pi \in \Sigma_{n}$, there is a coordinate map $\Gamma_{\pi}: \Lambda_{n} \rightarrow \mathcal{E}$ defined by $\Gamma_{\pi}(\lambda)=f_{(\pi, \lambda)}$. The definition of the map $f_{(\pi, \lambda)}$ in Equations (1)-(3) extends to vectors $\lambda \in \bar{\Lambda}_{n}$ by allowing some of the partition intervals $I_{i}$ to be degenerate. Thus, the coordinate maps extend to maps $\Gamma_{\pi}: \bar{\Lambda}_{n} \rightarrow \mathcal{E}$. The needed topological conditions essentially require the $\Gamma_{\pi}$ to be continuous parameterizations.

Theorem 1.1 Suppose that $\mathcal{E}$ has a topological group structure such that for every unpartitioned permutation $\pi \in \Sigma_{n}$, the coordinate map $\Gamma_{\pi}: \bar{\Lambda}_{n} \rightarrow \mathcal{E}$ is continuous and the restriction $\left.\Gamma_{\pi}\right|_{\Lambda_{n}}$ is a homeomorphism onto its image. Then an action $\mathbb{R} \rightarrow \mathcal{E}$ is continuous if and only if it is a rotation action.

Due to this result, the image in $\mathcal{E}$ of a rotation action will be referred to as a oneparameter subgroup of $\mathcal{E}$. Based on the classification in [15] of interval exchange centralizers, it is not hard to see that if two one-parameter subgroups commute, then they are simultaneously conjugate to subgroups of a common standard torus action. Thus, the group generated by two distinct commuting one-parameter subgroups is conjugate to the image of a two-dimensional subgroup of $\mathbb{T}^{n}$ under some standard torus action. However, the situation for noncommuting one-parameter subgroups appears to be quite different.

Conjecture 1.2 Let $F=\left\{f_{t}\right\}$ and $G=\left\{g_{s}\right\}$ be one-parameter subgroups of $\mathcal{E}$. If $F$ and $G$ do not commute, then the group $\langle F, G\rangle$ has elements that are not contained in any one-parameter subgroup of $\mathcal{E}$.

A consequence of this conjecture is the observation that up to conjugacy in $\mathcal{E}$, an interval exchange action of a connected Lie group must factor through a standard torus action.

A motivating example for this conjecture is the pair of one-parameter subgroups $F=\left\{r_{t}\right\}$ and $G=\left\{r_{s, \delta}\right\}$, where the map $r_{s, \delta}$ denotes a restricted rotation by $s \delta(\bmod \delta)$ supported on the interval $[0, \delta)$. It can be shown that certain products in $\langle F, G\rangle$, such as $h=r_{t} \circ r_{s, \delta}$ with sufficiently small $t$ and $s$, will have linear discontinuity growth; 
ie, if $D\left(h^{n}\right)$ denotes the number of discontinuities of $h^{n}$, then $D\left(h^{n}\right) \sim C n$ for some $C>0$. It is not difficult to see that interval exchanges with linear discontinuity growth cannot be in the image of a rotation action. Hence, by Theorem 1.1, the group $\langle F, G\rangle$ contains elements that do not lie on any one-parameter interval exchange group.

Acknowledgements The author is indebted to Professor John Franks for suggesting this topic of study and for many helpful conversations while completing this work. The author would like to thank the referee for many useful comments and for suggesting much important background information.

\section{Coordinates and topology of $\mathcal{E}$}

The restriction to unpartitioned permutations is suggested by the fact that such a permutation $\pi$ properly describes the discontinuities of the map $f_{(\pi, \lambda)}$.

Lemma $2.1 \pi \in \Sigma_{n}$ is unpartitioned if and only if, for any $\lambda \in \Lambda_{n}$, the interval exchange $f_{(\pi, \lambda)}$ is discontinuous, as a map $[0,1) \rightarrow[0,1)$, at precisely each of $\beta_{1}, \ldots, \beta_{n-1}$.

Proof If $\pi(j+1)=\pi(j)+1$ for some $j$, then for any $\lambda \in \Lambda_{n}$, the map $f_{(\pi, \lambda)}$ restricts to a translation on $I_{j} \cup I_{j+1}$. In particular, $f_{(\pi, \lambda)}$ is continuous at $\beta_{j}$.

Conversely, if $f=f_{(\pi, \lambda)}$ is continuous at $\beta_{j}$, then both $I_{j}$ and $I_{j+1}$ are translated the same distance by $f$. Consequently, $\pi(j+1)=\pi(j)+1$, which implies $\pi$ is not unpartitioned.

Proposition 2.2 For any interval exchange $f \in \mathcal{E}$, there exists a positive integer $n$, an unpartitioned $\pi \in \Sigma_{n}$, and $\lambda \in \Lambda_{n}$, all of which are unique, such that $f=f_{(\pi, \lambda)}$.

Proof To show the existence of $n, \pi$, and $\lambda$, let

$$
0<\beta_{1}<\beta_{2}<\cdots<\beta_{n-1}<1
$$

be the finite set of points in $(0,1)$ at which $f$ is discontinuous as a map $[0,1) \rightarrow[0,1)$; this defines $n$. Setting $\beta_{0}=0$ and $\beta_{n}=1$, define $\lambda \in \Lambda_{n}$ by

$$
\lambda_{j}=\beta_{j}-\beta_{j-1}, \quad j=1, \ldots, n .
$$

The permutation $\pi$ is defined by the reordering of the points $\beta_{i-1}$ induced by the map $f$. Thus, $\pi(i)=j$ if and only if $\#\left\{k: f\left(\beta_{k}\right)<f\left(\beta_{i-1}\right)\right\}+1=j$. By construction $f=f_{(\pi, \lambda)}$, and $\pi$ is unpartitioned by Lemma 2.1, since $f$ is discontinuous at precisely $\beta_{1}, \ldots, \beta_{n-1}$. The uniqueness of $\pi$ and $\lambda$ now follows since these data were constructed using intrinsic features of the transformation $f$. 
The most natural choice of a topology on $\mathcal{E}$ that satisfies the conditions of Theorem 1.1 is the $\mathrm{CW}$-complex topology induced by the cell maps $\Gamma_{\pi}$. It is not difficult to check that the group operations of $\mathcal{E}$ are continuous with respect to this topology. In particular, since the $\mathrm{CW}$-complex structure for $\mathcal{E}$ involves only countably many cells, the product $\mathcal{E} \times \mathcal{E}$ has a $\mathrm{CW}$-complex structure induced by the cell maps $\Gamma_{\pi} \times \Gamma_{\sigma}$ (see Hatcher [7, Proposition A-2, page 521]). In addition, a proof of Theorem 1.1 with respect to the CW-complex topology is not difficult (particularly given Lemma 3.1 and Proposition 3.2 below); this is due to the fact that in the cell topology any compact subset must be contained within finitely many cells.

However, the $\mathrm{CW}$ topology on $\mathcal{E}$ does not properly reflect the action of $\mathcal{E}$ on various function spaces defined over $\mathbb{T}$. For instance, consider the following sequence $f_{n}$ of

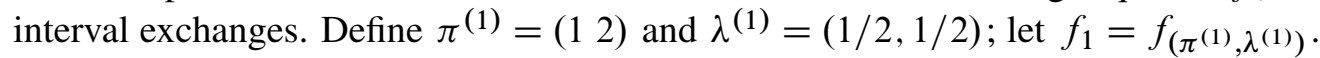
For integers $n \geq 2$, let

$$
\begin{aligned}
& \pi^{(n)}=(12) \cdot(34) \cdots((2 n-1)(2 n)) \cdot(2 n+1) \in \Sigma_{2 n+1}, \\
& \lambda^{(n)}=\left(\frac{1}{2^{n+1}}, \frac{1}{2^{n+1}}, \ldots, \frac{1}{2^{n+1}}, 1-\frac{n}{2^{n}}\right),
\end{aligned}
$$

and let $f_{n}=f_{\left(\pi^{(n)}, \lambda^{(n)}\right)}$; see Figure 4 .

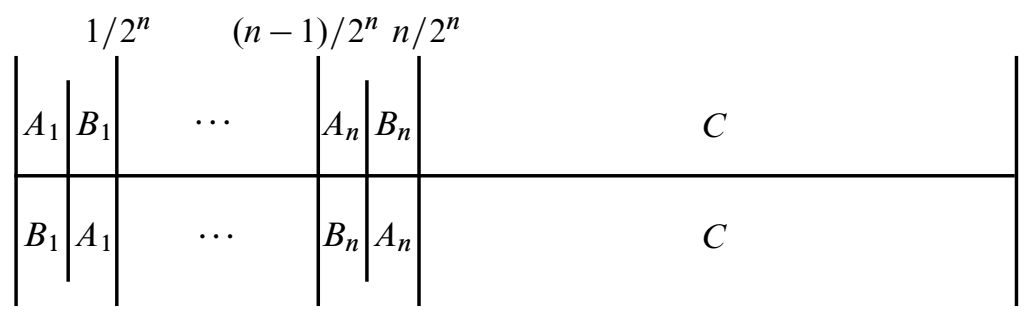

Figure 4: The map $f_{n}$

Since each element of $\left\{f_{n}\right\}$ is in a different cell, this set is closed in the CW topology of $\mathcal{E}$. In particular, it does not contain the identity as a limit point, even though the mappings $f_{n}$ converge uniformly to the identity.

The topology of uniform convergence is also not a suitable topology on $\mathcal{E}$ since the maps are not usually continuous. For instance, the sequence of maps $g_{n}=g_{\left(\sigma^{(n)}, \eta^{(n)}\right)}$ defined by

$$
\sigma^{(n)}=\left(\begin{array}{ll}
1 & 3
\end{array}\right) \in \Sigma_{4}, \quad \eta^{(n)}=\left(\frac{1}{2^{n}}, \frac{2^{n-1}-1}{2^{n}}, \frac{1}{2^{n}}, \frac{2^{n-1}-1}{2^{n}}\right)
$$

does not converge uniformly to the identity; see Figure 5. 


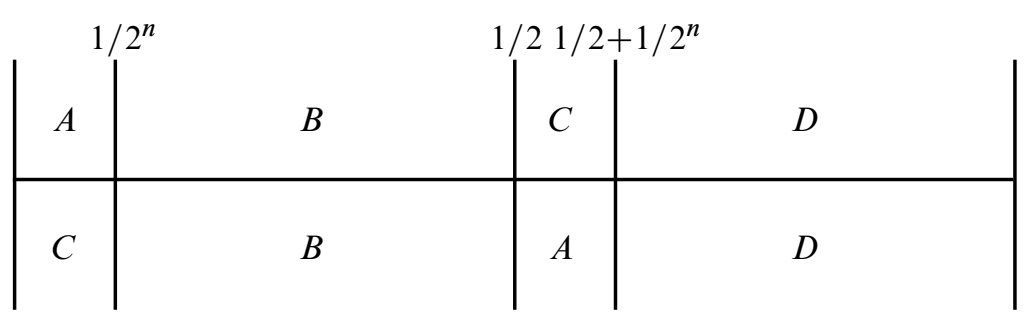

Figure 5: The map $g_{n}$

We now define a topological group structure on $\mathcal{E}$ in which both of the above sequences converge to the identity. Let $\rho$ denote the shortest-path metric on the circle $\mathbb{T}$, identified with $[0,1)$. Given $f, g \in \mathcal{E}$, define

$$
d(f, g)=\int_{\mathbb{T}} \rho(f(x), g(x)) d \mu(x),
$$

where $\mu$ denotes Lebesgue measure.

Proposition 2.3 The function $d$ is a metric on $\mathcal{E}$, and the metric space $(\mathcal{E}, d)$ is a topological group.

Proof The nonnegativity, symmetry, and triangle inequality for $d$ follow from the corresponding properties of the metric $\rho$. Moreover, note that $d(f, g)=0$ implies that $f(x)=g(x) \mu$-a.e., and two interval exchanges that coincide $\mu-$ a.e. must be identical. Thus $d$ is a metric.

To show that composition is continuous with respect to $d$, suppose there are convergent sequences $f_{n} \rightarrow f$ and $g_{n} \rightarrow g$. It suffices to estimate for all sufficiently large $n$ that $f_{n}\left(g_{n}(x)\right)$ is close to $f(g(x))$ outside of a set with small measure, since the $\mathbb{T}-$ metric $\rho$ is bounded on the exceptional set. To achieve this estimate, two comparisons can be made. First, for sufficiently large $n$, the $\mathbb{T}$-distance between $f_{n}\left(g_{n}(x)\right)$ and $f\left(g_{n}(x)\right)$ is small outside of a set with small measure since $d\left(f_{n}, f\right)$ is close to zero. Next, the distance between $f\left(g_{n}(x)\right)$ and $f(g(x))$ is small outside of a set with small measure since $d\left(g_{n}, g\right)$ is close to zero and since the map $f$ is a translation on most sufficiently small intervals. Thus, composition is continuous with respect to the metric $d$.

It remains to show that inversion in $\mathcal{E}$ is continuous. First, note that the metric $\rho$ is invariant under right translation in the group, since all interval exchange transformations preserve Lebesgue measure. Thus,

$$
d(f, \mathrm{id})=d\left(\mathrm{id}, f^{-1}\right) .
$$


Consequently, if $f_{n} \rightarrow$ id, then $f_{n}^{-1} \rightarrow$ id. Thus inversion is continuous at the identity. In general, if $f_{n} \rightarrow f$, the continuity of composition implies that $f^{-1} f_{n} \rightarrow$ id. But then $f_{n}^{-1} f \rightarrow \mathrm{id}$, and applying the continuity of composition again yields $f_{n}^{-1} \rightarrow f^{-1}$, as desired.

Proposition 2.4 For any unpartitioned permutation $\pi$, the map $\Gamma_{\pi}: \bar{\Lambda}_{n} \rightarrow \mathcal{E}$ is continuous with respect to the metric $d$. Consequently, the restriction $\left.\Gamma_{\pi}\right|_{\Lambda_{n}}$ is a homeomorphism onto its image.

Proof It has been shown in Proposition 2.2 that the restriction $\left.\Gamma_{\pi}\right|_{\Lambda_{n}}$ is injective. If the map $\Gamma_{\pi}$ is continuous, then the restriction $\left.\Gamma_{\pi}\right|_{\Lambda_{n}}$ is a homeomorphism onto its image due to the compactness of $\bar{\Lambda}_{n}$.

To show the continuity of $\Gamma_{\pi}: \bar{\Lambda}_{n} \rightarrow \mathcal{E}$, suppose that $\lambda^{(m)} \rightarrow \lambda$ in $\bar{\Lambda}_{n}$, and let $f^{(m)}$ and $f$ denote $\Gamma_{\pi}\left(\lambda^{(m)}\right)$ and $\Gamma_{\pi}(\lambda)$, respectively. Given some $\epsilon>0$, for all sufficiently large $m$, we have

$$
\left|\lambda_{j}-\lambda_{j}^{(m)}\right|<\frac{\epsilon}{n}, \quad j=1, \ldots, n .
$$

Comparing the difference between boundary points of the partition intervals of $f^{(m)}$ and $f$, we have

$$
\left|\beta_{j}-\beta_{j}^{(m)}\right|=\left|\sum_{k=1}^{j} \lambda_{k}-\sum_{k=1}^{j} \lambda_{k}^{(m)}\right| \leq \sum_{k=1}^{j}\left|\lambda_{k}-\lambda_{k}^{(m)}\right|<\epsilon .
$$

Thus, for sufficiently large $m$, the partition intervals $I_{j}$ and $I_{j}^{(m)}$ overlap up to a set of small measure. That is,

$$
\mu\left(I_{j} \backslash I_{j}^{(m)}\right)<2 \epsilon .
$$

Next, observe that the translation vectors $\omega^{(m)}=\Omega_{\pi}\left(\lambda^{(m)}\right)$ converge to $\omega=\Omega_{\pi}(\lambda)$, since the map $\Omega_{\pi}$ is linear. Thus, for all sufficiently large $m$,

$$
\left|\omega_{j}-\omega_{j}^{(m)}\right|<\epsilon .
$$

Therefore, $d\left(f, f^{(m)}\right)$ is equal to

$$
\begin{aligned}
& \sum_{j=1}^{n}\left(\int_{I_{j} \cap I_{j}^{(m)}} \rho\left(f x, f^{(m)} x\right) d \mu(x)+\int_{I_{j} \backslash I_{j}^{(m)}} \rho\left(f x, f^{(m)} x\right) d \mu(x)\right) \\
& \quad=\sum_{j=1}^{n} \int_{I_{j} \cap I_{j}^{(m)}} \rho\left(x+w_{j}, x+w_{j}^{(m)}\right) d \mu(x)+\sum_{j=1}^{n} \int_{I_{j} \backslash I_{j}^{(m)}} \rho\left(f x, f^{(m)} x\right) d \mu(x) .
\end{aligned}
$$

The first term in this last expression is bounded by $\epsilon$, since $\rho\left(x+w_{j}, x+w_{j}^{(m)}\right)<\epsilon$ on the sets $I_{j} \cap I_{j}^{(m)}$. The second term is bounded by $n \epsilon$, since $\rho \leq 1 / 2$ and 
$\mu\left(I_{j} \backslash I_{j}^{(m)}\right)<2 \epsilon$. Thus $d\left(f, f^{(m)}\right)<(n+1) \epsilon$ for all sufficiently large $m$, which proves that $\Gamma_{\pi}$ is continuous.

In addition to having the desired topological properties with respect to the coordinate maps $\Gamma_{\pi}$, the topology induced by the metric $d$ has another natural interpretation. Since all interval exchanges preserve Lebesgue measure on $[0,1)$, one may view the group $\mathcal{E}$ as a group of unitary operators on $L^{2}(\mathbb{T}, \mu)$.

Proposition 2.5 The topology induced on $\mathcal{E}$ by the metric $d$ coincides with the strong operator topology when $\mathcal{E}$ is viewed as a subgroup of $B\left(L^{2}(\mathbb{T}, \mu)\right)$.

Proof Let $\left\{f_{n}\right\}$ be a sequence in $\mathcal{E}$ and let $\left\{T_{n}\right\}$ be the corresponding sequence of unitary operators; similarly, let $f \in \mathcal{E}$ and let $T$ denote its corresponding operator.

First, suppose that $d\left(f_{n}, f\right)$ converges to zero, and let $\phi \in L^{2}(\mathbb{T})$ be any continuous function. As in the above arguments, having $d\left(f_{n}, f\right)$ close to zero means that $\rho\left(f_{n}(x), f(x)\right)<\delta$ on a set $A_{n}$ whose complement has measure approaching zero as $n$ increases. Using the uniform continuity of $\phi$, it follows that $\left|T_{n} \phi(x)-T \phi(x)\right|<\epsilon$ on $A_{n}$. Since $\phi$ is bounded by a constant $M$, the contribution to $\left\|T_{n} \phi-T \phi\right\|_{2}^{2}$ from points in the complement of $A_{n}$ is bounded by $4 M^{2} \mu\left(A_{n}^{c}\right)$, which approaches zero. Thus $T^{n}$ converges to $T$ in the strong operator topology.

Conversely, suppose $T^{n}$ converges strongly to $T$. Given $\epsilon>0$, partition $\mathbb{T}$ into intervals $I_{1}, I_{2}, \ldots, I_{M}$ such that $f$ is continuous on each interval $I_{j}$ and $\mu\left(I_{j}\right)<\epsilon$ for all $j$. Since $\left\|T_{n} \chi_{I_{j}}-T \chi_{I_{j}}\right\|_{2} \rightarrow 0$, there exists $N_{j}$ such that

$$
\mu\left(\left\{x \in I_{j}: f_{n}(x) \in f\left(I_{j}\right)\right\}\right)>(1-\epsilon) \mu\left(I_{j}\right)
$$

for all $n \geq N_{j}$. Since $f\left(I_{j}\right)$ is an interval, we have $\rho\left(f_{n}(x), f(x)\right)<\epsilon$ for any $x \in I_{j}$ such that $f_{n}(x) \in f\left(I_{j}\right)$. Let $N=\max \left\{N_{j}\right\}$. Then for any $n>N$, the set of points $x \in \mathbb{T}$ for which $\rho\left(f_{n}(x), f(x)\right)<\epsilon$ has measure greater than $(1-\epsilon)$. Thus $d\left(f_{n}, f\right)<\epsilon+(1 / 2) \epsilon$, which implies $d\left(f_{n}, f\right) \rightarrow 0$.

\section{Proof of the classification theorem}

In preparation for proving Theorem 1.1, it is useful to describe the dynamics of a rotation subgroup $F=\left\{f_{t}\right\}$ on $[0,1)$. Suppose $f_{t}=f_{([t \alpha], \lambda)}$, where $\alpha \in \mathbb{R}^{n}$ and $[t \alpha]$ is the equivalence class of $t \alpha$ in $\mathbb{T}^{n} \cong[0,1)^{n}$. See Equation (4) to recall the definition of $f_{([t \alpha], \lambda)}$. In this case, $f_{t}$ rotates each of the $\lambda$-partition intervals $I_{j}$ by $t \alpha_{j} \lambda_{j}\left(\bmod \lambda_{j}\right)$. The nontrivial orbits of the action are the intervals $I_{j}$ for which the rotation rate $\alpha_{j}$ is nonzero. 
In general, a rotation subgroup $F=\left\{f_{t}\right\}$ is conjugate in $\mathcal{E}$ to a subgroup $\left\{f_{([t \alpha], \lambda)}\right\}$ of a standard torus action; thus, $f_{t}=h f_{([t \alpha], \lambda)} h^{-1}$ for some $h \in \mathcal{E}$. The conjugacy $h$ may break a nontrivial $\left\{f_{([t \alpha], \lambda)}\right\}$-orbit into several intervals, but for each point $x$ in the interior of such an interval, the maps $f_{t}$ still locally translate $x$ by $t \alpha_{j} \lambda_{j}$. See an example in Figure 6.

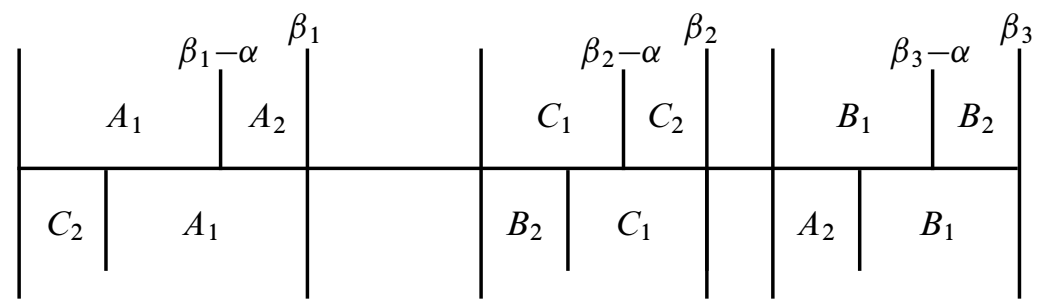

Figure 6: A map conjugate to a restricted rotation

To make a precise statement, let $\operatorname{Fix}(F)$ denote the set of global fixed points for a given one-parameter subgroup $F=\left\{f_{t}\right\}$. Let $\mathcal{P}$ denote the set algebra of all finite unions of half-open intervals $[a, b)$ in $[0,1)$.

Lemma 3.1 A one-parameter subgroup $F=\left\{f_{t}\right\}$ of $\mathcal{E}$ is a rotation subgroup if and only if $\operatorname{Fix}(F) \in \mathcal{P}$ and for all but finitely many $x \in[0,1)$, there exists $\alpha_{x} \in \mathbb{R}$ and $\epsilon_{x}>0$, such that

$$
f_{t}(x)=x+t \alpha_{x} \quad \text { if }|t|<\epsilon_{x} .
$$

Proof It is easy to see that if $F$ is conjugate to a rotation subgroup, then the action of $f_{t}$ satisfies the local condition stated in the lemma. In particular, if $f_{t}=h f_{([t \alpha], \lambda)} h^{-1}$ for all $t$, then the finite set of points that do not satisfy the condition is contained in the image under $h$ of the union of the set of discontinuities of $h$ and the set of partition interval endpoints induced by the length vector $\lambda$.

Conversely, suppose $\operatorname{Fix}(F) \in \mathcal{P}$ and $f_{t}$ is locally a rotation at all but finitely many points. Let $0=x_{0}<x_{1}<x_{2}<\cdots<x_{n}=1$ be the exceptional points, including all boundary points of $\operatorname{Fix}(F)$. Over all $x \in\left(x_{i-1}, x_{i}\right)$ the rotation speed $\alpha_{x}$ must be constant, since by definition it is locally constant. It remains to consider the behavior of $f_{t}$ at the exceptional points.

Consider the interval $I_{j}=\left[x_{j-1}, x_{j}\right)$ of length $\lambda_{j}$. Let $\alpha_{j}$ denote the constant rotation speed on the interior points of $I_{j}$. If $\alpha_{j}=0$, then $I_{j} \subseteq \operatorname{Fix}(F)$, so assume $\alpha_{j} \neq 0$. By 
replacing $t$ with $-t$, it can be assumed that $\alpha_{j}$ is positive. For sufficiently small nonnegative $t$ and for sufficiently small $\delta$ such that $0<\delta \ll \lambda_{j}$, the interval $\left(x_{j-1}, x_{j-1}+\delta\right)$ is translated by $t \alpha_{j}$ under $f_{t}$. Since the maps $f_{t}$ are all right-continuous at $x_{j-1}$, it follows that $f_{t}\left(x_{j-1}\right)=x_{j-1}+t \alpha_{j}$ for all sufficiently small nonnegative $t$.

In fact, the group $f_{t}$ acts (locally) on all of $\left(x_{j-1}, x_{j}\right)$ by translation by $t \alpha_{j}$; thus

$$
f_{t}\left(x_{j-1}\right)=x_{j-1}+t \alpha_{j} \text { for } 0 \leq t<\lambda_{j} / \alpha_{j}
$$

Consider what happens for $t=\lambda_{j} / \alpha_{j}$. First, suppose the interval $I_{j}$ is $f_{t}$-invariant. If $y=f_{\left(\lambda_{j} / \alpha_{j}\right)}\left(x_{j-1}\right)$ is in the interior of $I_{j}$, then $\left[y, x_{j}\right)$ is a periodic orbit properly contained in the orbit of $x_{j-1}$, which is impossible. Thus, $f_{\left(\lambda_{j} / \alpha_{j}\right)}\left(x_{j-1}\right)=x_{j-1}$, and the action of $f_{t}$ on $I_{j}$ is globally a rotation action.

In general, if $I_{j}$ is not invariant, suppose that $y$ is in the interior of some $I_{k}$, with $k \neq j$, since $y \in I_{j}$ would imply invariance. For small $t<0, f_{t}(y)$ is in $I_{k}$, since the $f_{t}$ locally act as a rotation on the interior of $I_{k}$. However, $y=f_{\left(\lambda_{j} / \alpha_{j}\right)}\left(x_{j-1}\right)$, and it is also the case that $f_{t}(y)$ is in $I_{j}$ for small $t<0$, which is a contradiction. Thus, $f_{\left(\lambda_{j} / \alpha_{j}\right)}\left(x_{j-1}\right)$ must be some other exceptional point $x_{k-1}$. The transformations $f_{t}$ all preserve Lebesgue measure, and by right-continuity $f_{t}\left(x_{k-1}\right)=x_{k-1}+t \alpha_{k}$ for small $t \geq 0$. Thus, if $f_{\left(\lambda_{j} / \alpha_{j}\right)}\left(x_{j-1}\right)=x_{k-1}$, then $\alpha_{j}=\alpha_{k}$. Consequently, the orbit of $x_{j-1}$ is a finite union of intervals $I_{k}$, each of which has the same rotation speed. After applying a suitable conjugacy, each invariant collection of these intervals may be reassembled into a single invariant subinterval on which the conjugate action is a standard rotation action.

It is possible to improve on the previous lemma's recharacterization of rotation actions. In particular, since all interval exchanges preserve Lebesgue measure on $\mathbb{T}$, the condition of a point $x$ having an orbit locally given by a rotation action can be weakened to the condition that $t \mapsto f_{t}(x)$ is continuous for $t$ in a neighborhood of zero. If $x$ satisfies this weaker condition, it is said to have a locally continuous orbit under $f_{t}$.

Proposition 3.2 A one-parameter subgroup $F=\left\{f_{t}\right\}$ is a rotation subgroup if and only if $\operatorname{Fix}(F) \in \mathcal{P}$ and for all but finitely many $x \in[0,1)$, the function $\mathbb{R} \rightarrow[0,1)$ defined by

$$
t \mapsto f_{t}(x)
$$

is continuous in some open neighborhood around $t=0$. 
Proof By applying the previous lemma, it suffices to show that if $x$ has a locally continuous orbit, then there exists $\alpha_{x}$, such that $f_{t}(x)=x+t \alpha_{x}$ for all $t$ in a neighborhood of zero. If $f_{t}(x)=x$ for all $t$ in a neighborhood, then $x$ is a global fixed point of the action, and $\alpha_{x}=0$ will suffice.

Suppose that $x$ is not a global fixed point, and assume that the orbit $t \mapsto f_{t}(x)$ is continuous for $t \in[-\epsilon, \epsilon]$. By reducing $\epsilon$ if necessary, it can be assumed that the function $t \mapsto f_{t}(x)$ is one-to-one on $[-\epsilon, \epsilon]$.

By reversing the parameter $t$, it can be assumed that $t \mapsto f_{t}(x)$ is increasing on $[-\epsilon, \epsilon]$. Define $\alpha \neq 0$ to satisfy

$$
f_{\epsilon}(x)=x+\epsilon \alpha .
$$

Next, for any $n \in \mathbb{N}$, consider the increasing sequence of points

$$
f_{-\epsilon}(x), f_{-(n-1) \epsilon / n}(x), \ldots, x, f_{\epsilon / n}(x), f_{2 \epsilon / n}(x), \ldots, f_{\epsilon}(x) .
$$

Since all $f_{t}$ preserve Lebesgue measure $\mu$,

$$
\mu\left(\left[f_{(j-1) \epsilon / n}(x), f_{j \epsilon / n}(x)\right)\right)=\mu\left(\left[f_{(k-1) \epsilon / n}(x), f_{k \epsilon / n}(x)\right)\right),
$$

for all $j$ and $k$ satisfying $-n<j, k \leq n$. Consequently,

$$
f_{j \epsilon / n}(x)=x+(j \epsilon / n) \alpha \quad \text { for all integers } j \text { such that }|j| \leq n .
$$

Thus, $f_{t}(x)=x+t \alpha$ for a dense set of $t \in[-\epsilon, \epsilon]$, and by continuity of the orbit this holds at all $t \in[-\epsilon, \epsilon]$.

Therefore, to prove Theorem 1.1 it suffices to prove the following:

Proposition 3.3 If $F=\left\{f_{t}\right\}$ is a continuous one-parameter subgroup of $\mathcal{E}$, then all but finitely many $x \in[0,1)$ have locally continuous orbits and $\operatorname{Fix}(F) \in \mathcal{P}$.

Before giving a proof of this proposition, it will be shown that for any one-parameter subgroup $\left\{f_{t}\right\}$, the number of discontinuities of $f_{t}$ is bounded over all $t$ in some neighborhood of zero. Define the function $\delta: \mathcal{E} \rightarrow \mathbb{N}$ by

$$
\delta(f)=n \text {, where } f=f_{(\pi, \lambda)} \text { for (unique) unpartitioned } \pi \in \Sigma_{n}, \lambda \in \Lambda_{n} .
$$

Equivalently, $\delta(f)$ returns the number of discontinuities of $f$, considered as a map $[0,1) \rightarrow[0,1)$, counting 0 as a discontinuity. Note that $\delta(f)=n$ if and only if $f$ is in the image of the interior of the (n-1)-dimensional simplex $\bar{\Lambda}_{n}$ under the parametrization $\Gamma_{\pi}$, for some unique unpartitioned $\pi \in \Sigma_{n}$. The simplex $\bar{\Lambda}_{n}$ is 
compact, and the number of unpartitioned permutations in $\Sigma_{k}$ for $k \leq n$ is finite. Since the parametrizations $\Gamma_{\pi}$ are continuous, the sets

$$
K_{n}=\{f \in \mathcal{E}: \delta(f) \leq n\}
$$

are compact. Therefore, if $\delta(f)=n$, then $f$ is in the interior of $\mathcal{E} \backslash K_{n-1}$. Consequently, for all $g$ in some neighborhood of $f, \delta(g) \geq \delta(f)$; in other words, the function $\delta$ is lower semicontinuous.

Lemma 3.4 For any continuous one-parameter subgroup $F=f_{t}$, the function $t \mapsto$ $\delta\left(f_{t}\right)$ is bounded on any compact subset of $\mathbb{R}$.

Proof Since $f_{s+t}=f_{s} \circ f_{t}$ for all $s, t \in \mathbb{R}$, it follows that

$$
\delta\left(f_{s+t}\right) \leq \delta\left(f_{s}\right)+\delta\left(f_{t}\right), \quad s, t \in \mathbb{R} .
$$

This inequality records the fact that a composition of two interval exchange maps cannot have more discontinuities than occur over both of its factors. From this inequality, it also follows that

$$
\delta\left(f_{s+t}\right) \geq\left|\delta\left(f_{s}\right)-\delta\left(f_{t}\right)\right|, \quad s, t \in \mathbb{R} .
$$

By (5), if $\delta\left(f_{t}\right)$ is bounded for $t \in[-\epsilon, \epsilon]$, then $\delta\left(f_{t}\right)$ is bounded on all compact subsets. Thus, if $\delta\left(f_{t}\right)$ is unbounded on some compact subset, then $\delta\left(f_{t}\right)$ is unbounded in any neighborhood of zero. In fact, the inequality (6) further implies that $\delta\left(f_{t}\right)$ is unbounded in any neighborhood of any $t \in \mathbb{R}$.

This local unboundedness and the semicontinuity of $\delta$ cannot coexist. To derive a contradiction, suppose that $\delta\left(f_{t}\right)$ is unbounded in any neighborhood of any $t$. Let

$$
A_{n}=\left\{t \in \mathbb{R}: \delta\left(f_{t}\right) \leq n\right\} .
$$

By the lower semicontinuity of $\delta$, the sets $A_{n}$ are closed, and their complements

$$
B_{n}=\{t \in \mathbb{R}: \delta(t)>n\}
$$

are open. If $\delta$ is locally unbounded at every point, each set $B_{n}$ is dense in $\mathbb{R}$. However,

$$
\bigcap B_{n}=\left\{t \in \mathbb{R}: \delta\left(f_{t}\right)>n \text { for all } n \in \mathbb{N}\right\}=\varnothing
$$

which is a contradiction by the Baire Category Theorem. Thus, $\delta\left(f_{t}\right)$ must be bounded on any compact subset of $\mathbb{R}$. 


\section{Proof of Proposition 3.3 Applying Lemma 3.4, let}

$$
n=\max \left\{\delta\left(f_{t}\right): t \in[-1,1]\right\} .
$$

By the lower semicontinuity of $\delta$, the set $\left\{t \in[-1,1]: \delta\left(f_{t}\right)=n\right\}$ is relatively open in $[-1,1]$. Therefore, there exists some $t_{0} \in(-1,1)$ and $\epsilon>0$, such that $\delta\left(f_{t}\right)=n$ for $t \in\left(t_{0}-\epsilon, t_{0}+\epsilon\right)$. Let $\pi \in \Sigma_{n}$ be the unique unpartitioned permutation such that $f_{t_{0}} \in \Gamma_{\pi}\left(\Lambda_{n}\right)$. By Proposition 2.2, the sets $\Gamma_{\sigma}\left(\Lambda_{n}\right)$ are pairwise disjoint as $\sigma$ ranges over $\Sigma_{n}^{\prime}$, the set of unpartitioned permutations in $\Sigma_{n}$. Since

$$
f_{t_{0}} \in \mathcal{E} \backslash\left(\bigcup_{\sigma \in \Sigma_{n}^{\prime} \backslash\{\pi\}} \Gamma_{\sigma}\left(\bar{\Lambda}_{n}\right)\right)
$$

and the sets $\Gamma_{\sigma}\left(\bar{\Lambda}_{n}\right)$ are compact, it follows that $f_{t_{0}}$ is actually in the interior of the set denoted above. Consequently, after possibly replacing $\epsilon$ by a smaller value, it follows that $f_{t} \in \Gamma_{\pi}\left(\Lambda_{n}\right)$ for all $t \in\left(t_{0}-\epsilon, t_{0}+\epsilon\right)$.

In this situation, it can be seen that the paths

$$
t \mapsto f_{t}(x)
$$

are continuous in a neighborhood of $t_{0}$ for all but finitely many points, namely the discontinuity points of $f_{t_{0}}$. For $t \in\left(t_{0}-\epsilon, t_{0}+\epsilon\right)$ let $\lambda^{(t)} \in \Lambda_{n}$ be such that

$$
f_{t}=\Gamma_{\pi}\left(\lambda^{(t)}\right),
$$

where the $\lambda^{(t)}$ vary continuously in $\Lambda_{n}$. Thus, if $x$ is an interior point of the interval $I_{j}$ induced by $f_{t_{0}}$, then

$$
t \mapsto f_{t}(x)=x+\Omega_{\pi}\left(\lambda^{(t)}\right)_{j}
$$

is continuous in a neighborhood of $t_{0}$. Since $f_{-t_{0}}$ is continuous at all but a finite number of points, the path $t \mapsto f_{t}(x)$ is continuous in a neighborhood of zero for all but finitely many points.

It remains to consider the set of global fixed points for $f_{t}$. As before, define $\beta_{j}^{(t)}$ in terms of $\lambda^{(t)}$ and let $I_{j}^{(t)}=\left[\beta_{j-1}^{(t)}, \beta_{j}^{(t)}\right)$. Suppose the interior of $I_{j}^{\left(t_{0}\right)}$ contains a global fixed point $x$. Then for all $t$ in some $\left(t_{0}-\epsilon, t_{0}+\epsilon\right)$, the point $x$ is located in the interval $I_{j}^{(t)}$. Thus, for each $t$ in $\left(t_{0}-\epsilon, t_{0}+\epsilon\right)$, the interval $I_{j}^{(t)}$ is fixed by $f_{t}$. In addition, the intervals $I_{j-1}^{(t)}$ and $I_{j+1}^{(t)}$ cannot be fixed by $f_{t}$, since otherwise $\pi$ would be partitioned. As a result, the boundary points $\beta_{j-1}^{(t)}$ and $\beta_{j}^{(t)}$ must be constant over $t \in\left(t_{0}-\epsilon, t_{0}+\epsilon\right)$, since otherwise there would be points fixed by $f_{t}$ for $t$ in some nonempty, proper open subset of $\mathbb{R}$, which is impossible. Thus, the set $\operatorname{Fix}(F)$ of global fixed points for $f_{t}$ is a finite union of intervals $I_{j}^{\left(t_{0}\right)}$, which implies that $\operatorname{Fix}(F)$ is a member of $\mathcal{P}$. 


\section{References}

[1] A Avila, G Forni, Weak mixing for interval exchange transformations and translation flows, Ann. of Math. (2) 165 (2007) 637-664 MR2299743

[2] C Bleak, A geometric classification of some solvable groups of homeomorphisms, J. Lond. Math. Soc. (2) 78 (2008) 352-372 MR2439629

[3] L Burslem, A Wilkinson, Global rigidity of solvable group actions on $S^{1}$, Geom. Topol. 8 (2004) 877-924 MR2087072

[4] B Farb, P Shalen, Groups of real-analytic diffeomorphisms of the circle, Ergodic Theory Dynam. Systems 22 (2002) 835-844 MR1908556

[5] J Franks, Distortion in groups of circle and surface diffeomorphisms, from: "Dynamique des difféomorphismes conservatifs des surfaces: un point de vue topologique", Panor. Synthèses 21, Soc. Math. France (2006) 35-52 MR2288284

[6] M Gromov, Groups of polynomial growth and expanding maps, Inst. Hautes Études Sci. Publ. Math. (1981) 53-73 MR623534

[7] A Hatcher, Algebraic topology, Cambridge Univ. Press (2002) MR1867354

[8] A Katok, Interval exchange transformations and some special flows are not mixing, Israel J. Math. 35 (1980) 301-310 MR594335

[9] M Keane, Interval exchange transformations, Math. Z. 141 (1975) 25-31 MR0357739

[10] M Keane, Non-ergodic interval exchange transformations, Israel J. Math. 26 (1977) 188-196 MR0435353

[11] G Margulis, Free subgroups of the homeomorphism group of the circle, C. R. Acad. Sci. Paris Sér. I Math. 331 (2000) 669-674 MR1797749

[12] H Masur, Interval exchange transformations and measured foliations, Ann. of Math. (2) 115 (1982) 169-200 MR644018

[13] A Navas, Groupes résolubles de difféomorphismes de l'intervalle, du cercle et de la droite, Bull. Braz. Math. Soc. (N.S.) 35 (2004) 13-50 MR2057043

[14] A Navas, Growth of groups and diffeomorphisms of the interval, Geom. Funct. Anal. 18 (2008) 988-1028 MR2439001

[15] C F Novak, Discontinuity-growth of interval-exchange maps, J. Mod. Dyn. 3 (2009) 379-405 MR2538474

[16] G Rauzy, Échanges d'intervalles et transformations induites, Acta Arith. 34 (1979) 315-328 MR543205

[17] W A Veech, Interval exchange transformations, J. Analyse Math. 33 (1978) 222-272 MR516048

[18] W A Veech, Gauss measures for transformations on the space of interval exchange maps, Ann. of Math. (2) 115 (1982) 201-242 MR644019 
[19] M Viana, Ergodic theory of interval exchange maps, Rev. Mat. Complut. 19 (2006) 7-100 MR2219821

[20] A Zorich, Flat surfaces, from: "Frontiers in number theory, physics, and geometry. I", (P Cartier, B Julia, P Moussa, P Vanhove, editors), Springer, Berlin (2006) 437-583 MR2261104

Department of Mathematics and Statistics, The University of Michigan-Dearborn 4901 Evergreen Road, Dearborn, MI 48128 USA

cfnovak@umd.umich.edu

http://www-personal. umd.umich.edu/ cfnovak/Site/Homepage.html

Received: 20 February 2010 